\title{
ZEITSCHRIFT FUR
}

\section{ANGEWANDTE GEOLOGIE}

HERAUSGEGEBEN VON

DER STAATLICHEN GEOLOGISCHEN KOMMISSION

UND DER ZENTRALEN VORRATSKOMMISSION

DER DEUTSCHEN DEMOKRATISCHEN REPUBLIK
AUS DEM INHALT

\section{J. Pomper}

Erkundung und Bemusterung von Rohstoffen für Portlandzement

M. Dlabaẽ

Erdölakkumulation im Wiener Becken

W. I. Smirnow

Probleme der Metallogenese von Geosynklinalen

E. Lewien \& H. Treppschuh

Über die Festlegung von Rohstoffkonditionen für die verschiedenen Stadien der geologischen Erkundung

K. Kauter

Zum Rohstoff Helium

G. Herzog

Zusammenhang zwischen Senkungserscheinungen und Grundwasserchemismus in Bad Frankenhausen

A. Lenk

Ermittlung der wahren Mächtigkeit aus Bohrungen beliebiger Lage 